\title{
Pan-Pim Kinase Inhibitor AZD1208 Suppresses Tumor Growth and Synergistically Interacts with Akt Inhibition in Gastric Cancer Cells
}

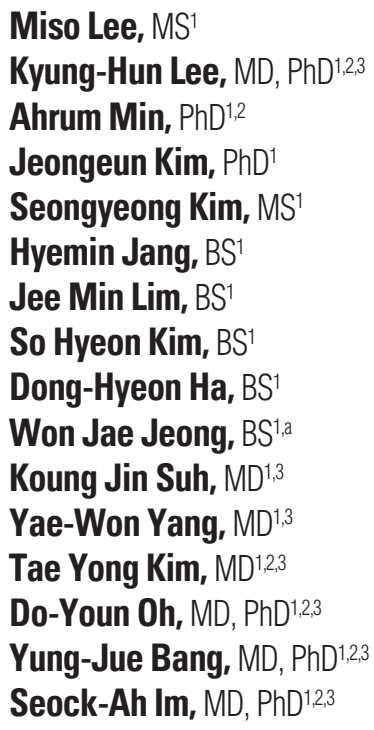

${ }^{1}$ Cancer Research Institute, Seoul National University, Seoul, ${ }^{2}$ Biomedical Research Institute, Seoul National University Hospital, Seoul, ${ }^{3}$ Department of Internal Medicine, Seoul National University College of Medicine, Seoul, Korea

Correspondence: Seock-Ah Im, MD, PhD Department of Internal Medicine,

Seoul National University College of Medicine, 101 Daehak-ro, Jongno-gu, Seoul 03080, Korea Tel: 82-2-2072-0850

Fax: 82-2-762-9662

E-mail: moisa@snu.ac.kr

Received July 17, 2017

Accepted May 30, 2018

Published Online June 6, 2018

aPresent address: University of California, Berkeley, CA, USA

\section{Purpose}

Pim kinases are highly conserved serine/threonine kinases, and different expression patterns of each isoform (Pim-1, Pim-2, and Pim-3) have been observed in various types of human cancers, including gastric cancer. AZD1208 is a potent and selective inhibitor that affects all three isoforms of Pim. We investigated the effects of AZD1208 as a single agent and in combination with an Akt inhibitor in gastric cancer cells.

\section{Materials and Methods}

The antitumor activity of AZD1208 with/without an Akt inhibitor was evaluated in a large panel of gastric cancer cell lines through growth inhibition assays. The underlying mechanism was also examined by western blotting, immunofluorescence assay, and cell cycle analysis.

\section{Results}

AZD1208 treatment decreased gastric cancer cell proliferation rates and induced autophagy only in long-term culture systems. Light chain 3B (LC3B), a marker of autophagy, was increased in sensitive cells in a dose-dependent manner with AZD1208 treatment, which suggested that the growth inhibition effect of AZD1208 was achieved through autophagy, not apoptosis. Moreover, we found that cells damaged by Pim inhibition were repaired by activation of the DNA damage repair pathway, which promoted cell survival and led the cells to become resistant to AZD1208. We also confirmed that the combination of an Akt inhibitor with AZD1208 produced a highly synergistic effect in gastric cancer cell lines.

\section{Conclusion}

Treatment with AZD1208 alone induced considerable cell death through autophagy in gastric cancer cells. Moreover, the combination of AZD1208 with an Akt inhibitor showed synergistic antitumor effects through regulation of the DNA damage repair pathway.

Key words

AZD1208, Pim kinase inhibitor, Autophagy, Akt, Stomach neoplasms 


\section{Introduction}

Pim kinases are a class of constitutively active serine/ threonine kinases consisting of three highly homologous members: Pim-1, Pim-2, and Pim-3. These factors were discovered as proviral insertion sites of the Moloney murine leukemia virus associated with the development of T-cell lymphomas. Members of this kinase family have been implicated in several biological processes, including cell survival, proliferation, and differentiation [1]. Pim kinase expression is mainly regulated by transcription factors, such as Janus kinase/Signal transducer and activator of transcription (JAK/STAT); the nuclear factor $\mathrm{kB}$, pathway; and ubiquitylation with subsequent proteasomal degradation at the post-translational level [2].

Pim kinases modulate cell proliferation by phosphorylating cell cycle regulators, such as p21, p27, Cdc25A, and Cdc25C, and mediate survival signal pathways by phosphorylating the Bcl-2 antagonist of cell death (Bad). Furthermore, these kinases have been found to induce phosphorylation of $4 \mathrm{E}-$ binding protein 1 (4E-BP1), which permits protein synthesis by $5^{\prime}$ cap-dependent translation [3]. Mechanistic studies have revealed that high expression levels of Pim are associated with tumorigenesis and cancer progression.

The expression level of each Pim isoform varies among tumor types [3]. More recently, the Pim-1 and Pim-3 levels have been found to be increased and predictive of poor prognosis in gastric cancer [4,5]. In particular, there is a relationship between aberrant expression of Pims and aggressive types of gastric cancer. Previous studies have reported that Pim-3 expression is greater in a tissue section of gastric adenoma compared to non-cancerous mucosa and is higher in intestinal-type gastric carcinoma than in diffuse-type gastric carcinoma. Interestingly, Pim-3 is expressed at a higher rate in metastatic sites than in primary sites of gastric carcinoma, and consequently, there is a lower survival rate of patients with Pim-3-positive gastric cancer [5]. Furthermore, several studies have also reported that the JAK/STAT signaling pathway, which directly modulates the expression levels of Pim kinases, is hyper-activated in gastric cancer [6]. Thus, Pim kinases have become a promising therapeutic target for the treatment of gastric cancer. However, it is well-known that Pim isoforms have compensatory effects, allowing them to maintain each other's functions [1]. For this reason, most Pim inhibitors are designed to inhibit all Pim isoforms; however, the effects of pan-Pim inhibitors and their underlying mechanisms have not been adequately explained in gastric cancer.

Dysregulation of the phosphatidylinositide 3-kinase (PI3K) / Akt pathway is commonly found in many types of human cancer, including gastric cancer [7]. Therefore, inhibition of this pathway is an attractive therapeutic target for treating gastric cancer. Although small molecule inhibitors that target Akt have been developed, due to the activation of compensatory signaling pathways following Akt inhibition, there have been many efforts to improve the effects of Akt inhibitors through combination strategies [8-10]. Previous studies have reported that resistance to Akt inhibition is mainly induced by mammalian target of rapamycin (mTOR) activation [11]. There have been many cases suggesting a treatment strategy of using mTOR and Akt inhibition; however, tolerability issues have been limiting. Pim and Akt kinases possess independent signaling pathways, but overlap in several areas. For example, PRAS40 activity is regulated by Pim kinases independent of Akt activation, but both directly regulate proapoptotic molecules, such as the Bcl-2 protein Bad $[12,13]$. Therefore, an inhibitory combination of Pim and Akt is expected to overcome Akt resistance by regulating mTOR activity. Furthermore, up-regulation of receptor tyrosine kinases by Pim induce resistance to Akt inhibitors [14]. Therefore, simultaneous inhibition of Pim and the Akt pathway could be a potent anticancer therapeutic strategy [15].

In the present study, the antitumor activity of the pan-Pim inhibitor AZD1208 [16], as well as its underlying mechanism, was evaluated in a large panel of gastric cancer cell lines. Subsequently, we explored the role of AZD1208 as a sensitizer when administered in combination with the Akt inhibitor AZD5363. Here, we show for the first time that treatment with AZD1208 alone exerts cytotoxic effects by inducing autophagy. This is the first report to show that inhibition of Pim kinases can suppress the growth of gastric cancer cells and that the DNA damage response is related to the sensitivity to AZD1208. We also show the therapeutic significance of dual inhibition of the Pim and Akt pathways, which synergistically decrease cell viability and improve drug efficacy in human gastric cancer cells.

\section{Materials and Methods}

\section{Reagents}

AZD1208, a Pim inhibitor [16,17], and AZD5363, an Akt inhibitor [18], were kindly provided by AstraZeneca (Macclesfield, Cheshire, UK). The chemical structure of AZD1208 is shown in Fig. 1A. 3-Methyladenine (3-MA) was purchased from Sigma-Aldrich (St. Louis, MO), and N-benzyloxycarbonyl-Val-Ala-Asp(O-Me) fluoromethyl ketone (z-VADfmk) was obtained from R\&D Systems (Minneapolis, MN). 


\section{Cell lines and cell culture}

Human gastric cancer cell lines (SNU-1, -5, -16, 216, -484, $-601,-620,-638,-668,-719$, AGS, MKN45, KATO-III, and N87) were purchased from the Korean Cell Line Bank (Seoul, Korea). The identities of the cell lines were authenticated by DNA fingerprinting analysis [19]. All cell lines were banked and passaged for less than 6 months before use. The cells were cultured in RPMI 1640 medium (Thermo Fisher Scientific Inc., Waltham, MA) supplemented with $10 \%$ fetal bovine serum (Welgene, Daegu, Korea) and $10 \mu \mathrm{g} / \mathrm{mL}$ gentamicin (Cellgro, Manassas, VA) at $37^{\circ} \mathrm{C}$ and $5 \% \mathrm{CO}_{2}$.

\section{Cell growth inhibition assay}

Cells (2-3×10 in $100 \mu \mathrm{L} /$ well) were seeded in 96-well plates (TPP, Trasadingen, Switzerland) and incubated overnight at $37^{\circ} \mathrm{C}$ and $5 \% \mathrm{CO}_{2}$. The cells were then exposed to increasing concentrations of AZD1208 (range, 0.001 to $10 \mu \mathrm{mol} / \mathrm{L}$ ) or dimethyl sulfoxide (DMSO; Sigma-Aldrich) for 5 days. After treatment, $50 \mu \mathrm{L}$ of a 3-(4,5-dimethylthiazol-2-yl)-2,5-diphenyltetrazolim bromide (MTT) solution (Sigma-Aldrich) was added to each well, and the plates were incubated for 4 hours at $37^{\circ} \mathrm{C}$ before the media was removed. After dissolving formazan crystals with $150 \mu \mathrm{L}$ of DMSO, the absorbance of each well was measured at $540 \mathrm{~nm}$ with a VersaMax microplate reader (Molecular Devices, Sunnyvale, CA). The absorbance and $\mathrm{IC}_{50}$ of the Pim inhibitor were analyzed using SigmaPlot software (SPSS Inc., Chicago, IL). Six replicate wells were included for each analysis, and at least three independent experiments were performed.

\section{Colony formation assay}

For the colony formation assay (CFA), cells were seeded into 6-well plates and incubated for 48 hours at $37^{\circ} \mathrm{C}$ in $5 \%$ $\mathrm{CO}_{2}$. Next, the cells were treated with various concentrations $(0.1,0.5,1,2.5$, and $5 \mu \mathrm{mol} / \mathrm{L})$ of AZD1208 alone or in combination with $100 \mathrm{nmol} / \mathrm{L}$ AZD5363 every 3 days. The cells were cultured until colonies formed (14 days). The colonies were stained with a $0.1 \%$ Coomassie blue solution (SigmaAldrich) and counted using a GelCount automatic plate scanner (Oxford Optronics GelCount, Oxford, UK). The cell survival rate and $\mathrm{IC}_{50}$ of AZD1208 were determined using SigmaPlot software (SPSS Inc.).

\section{Pyrosequencing analysis}

Target-specific primers were designed for pyrosequencing analysis as follows: PIK3CA-E453K forward, 5'-biotin-GCTTTGAATCTTTGGCCAGTAC-3'; PIK3CA-E453K reverse, 5' TCCAGTAACACCAATAGGGTTCAG-3'; PIK3CA-E542K and -E545K forward, $5^{\prime}$-biotin-CAGCTCAAAGCAATTTCTACACG-3'; PIK3CA-E542K and -E545K reverse, $5^{\prime}$-GCACTTACCTGTGACTCCATAGAA-3'; ${ }^{\prime}$ PIK3CA-H1047R forward, 5'-biotin-TTCGAAAGACCCTAGCCTTAGAT-3'; PIK3CAH1047R reverse, 5' -TGCTGTTTAATTGTGTGGAAGATC-3'. For PIK3CA-E453K, E542K, E545K, and H1047R, the pyrosequencing primers $5^{\prime}$-AGGGTTCAGCAAATCT- $3^{\prime}$, $5^{\prime}$-TTCTCCTGCTCAGTGAT- ${ }^{\prime}, 5^{\prime}$-CCATAGAAAATCTTTCTCCT$3^{\prime}$, and $5^{\prime}$-TGCTGTTTAATTGTGTGGAAGATC- ${ }^{\prime}{ }^{\prime}$ were used, respectively. Single-stranded biotinylated PCR products were processed for pyrosequencing analysis according to the manufacturer's standard protocol (PyroMark Q96 ID, Qiagen, Hilden, Germany).

\section{Reverse transcription-polymerase chain reaction and real-time polymerase chain reaction}

Total RNA was isolated using TRI reagent (Molecular Research Center, Cincinnati, $\mathrm{OH}$ ) according to the manufacturer's instructions. cDNA was synthesized by reverse transcription polymerase chain reaction (RT-PCR) with ImPromII reverse transcriptase (Promega, Madison, WI) and amplified using AmpliTaq Gold DNA polymerase (Applied Biosystems, Carlsbad, CA) with gene-specific primers. Quantitative real-time polymerase chain reaction (qRT-PCR) was conducted using an iCycler iQ detection system (Bio-Rad Laboratories, Inc., Hercules, CA) with SYBR Green. Data for all samples were normalized relative to actin cDNA. The sequences of the primers used for RT-PCR and qRT-PCR are listed in S1 Table. cDNA was synthesized at least three times from three independent sets of samples, and all PCR analyses were performed in triplicate.

\section{Western blot analysis}

Proteins were extracted, and equal amounts of protein were separated on 5\%-15\% sodium dodecyl sulfate-polyacrylamide gels as previously described [20]. Separated proteins were transferred onto nitrocellulose membranes, and the blots were probed with primary antibodies $(1: 1,000 \mathrm{dilu}-$ tion) overnight at $4^{\circ} \mathrm{C}$. Antibodies specific for the following factors were used: Pim-1 (sc-13513, Santa Cruz Biology Technology, Santa Cruz, CA), Pim-2 (CST 4730, Cell Signaling Technology, Beverly, MA), Pim-3 (CST 4165), phosphorylated (p)Bad Ser112 (CST 9296), Bad (CST 9292), p4EBP1 Ser65 (CST 9451), 4EBP1 (CST 9452), pS6K (CST 2211), S6K (CST 2217), Bcl-xL (sc-1690), Mcl-1 (sc-819), PARP (BD 556494), caspase-3 (CST 9662), light chain 3B (LC3B; CST 3868), Beclin-1 (ab51031), pATM Ser1981 (CST 4526), ATM (sc-23921), pChk2 Thr68 (CST 2661), Chk2 (sc-5278), pAkt Ser473 (CST 9271), Akt (CST 9272), pPRAS40 Thr246 (CST 2997), PRAS40 (CST 2691), and $\alpha$-tubulin (Sigma-Aldrich). Anti- 
body binding was detected using an enhanced chemiluminescence system according to the manufacturer's protocol (Amersham Biosciences, Piscataway, NJ).

\section{Cell-cycle analysis}

Cells treated with AZD1208 were harvested, fixed with cold $70 \%$ ethanol, and then stored at $-20^{\circ} \mathrm{C}$ for at least 24 hours. Next, the cells were washed in phosphate buffered saline (PBS), incubated with $10 \mu \mathrm{g} / \mathrm{mL}$ RNase A (SigmaAldrich) at $37^{\circ} \mathrm{C}$ for 20 minutes, and stained with $20 \mu \mathrm{g} / \mathrm{mL}$ propidium iodide (Sigma-Aldrich). The DNA levels of the cells (10,000 cells per group) were quantified using a FACS Calibur flow cytometer (BD Biosciences, San Jose, CA).

\section{Annexin $\mathrm{V}$ binding assay}

After the cells were exposed to AZD1208, the degree of apoptosis was assessed using the Annexin V binding assay according to the protocols of the manufacturer (BD PharMingen, San Jose, CA). The harvested cell suspension was then incubated with Annexin V for 15 minutes at room temperature in the dark and analyzed by flow cytometry.

\section{Immunofluorescence assay}

Cells were plated on $0.01 \%$ poly-L-Lysine (Sigma Aldrich)coated coverslips, transfected with GFP-labeled LC3B and treated with 1 or $5 \mu \mathrm{M}$ AZD1208. After 5 days, the coverslips were rinsed once in PBS $\left(37^{\circ} \mathrm{C}\right)$, fixed in $3.7 \%$ paraformaldehyde for 10 minutes, permeabilized with PBS-T $(0.5 \%$ TritonX-100 in PBS) for 5 minutes, and incubated with primary antibodies for 24 hours at $4^{\circ} \mathrm{C}$. The primary antibodies used in this study included anti-LC3B, -pChk2, and anti- $\gamma \mathrm{H} 2 \mathrm{AX}$ at a dilution of 1:50. The coverslips were rinsed three times for 10 minutes in PBS, followed by incubation with the appropriate fluorophore-conjugated secondary antibody (Invitrogen, Carlsbad, CA). The cells were counterstained with DAPI (300 nM, Invitrogen), and the coverslips were mounted onto slides using Faramount aqueous mounting medium (Dako, Glostrup, Denmark). Immunofluorescence was visualized using a Nikon A1 confocal laser scanning microscope (Nikon, Tokyo, Japan).

\section{In vivo study}

The animal experiments were carried out at the animal facility of Seoul National University (Seoul, Korea) according to the institutional guidelines with prior approval from the Institutional Animal Care and Use Committee. Six-week-old female BALB/c nude mice purchasing from Central Lab Animal Inc. (Seoul, Korea) were used to test the in vivo activ- ities of AZD1208. The mice were injected subcutaneously in the right flank with $7 \times 10^{7}$ of SNU-638 cells in $100 \mu \mathrm{L}$ of PBS. After implantation of the tumor cells, the tumor sizes were measured every other day using calipers, and the body weight of each mouse was determined twice per week. The mice were randomly divided into two groups (five mice per group) when tumor volumes reached $200 \mathrm{~mm}^{3}$, and $45 \mathrm{mg}$ / $\mathrm{kg}$ of AZD1208 were administered via oral gavage once daily for 28 consecutive days. The control group was treated with vehicle alone ( $1 \mathrm{mM}$ histidine, $130 \mathrm{mM}$ Glycine, $5 \%$ sucrose in water). Tumor volume was examined not only during treatment but also after treatment was ceased and calculated using the following formula: (width ${ }^{2} \times$ height) $/ 2$. The mice were sacrificed with $\mathrm{CO}_{2}$ at the end of the observation period, and tumors were excised for further analysis.

\section{Immunohistochemistry}

Paraffin-embedded xenograft tumor tissues were deparaffinized with xylene and rehydrate with graded ethanol. Immunohistochemistry studies for Ki- 67 were conducted by using the anti-rabbit polyclonal antibody against Ki-67 (dilution of 1:100, GeneTex, Irvine, CA) and a terminal deoxynucleotidyl transferase-mediated dUTP nick end labeling assay performed to measure the apoptosis of xenografts using ApopTag In situ Apoptosis Detection Kit (Chemicon International, Temecula, CA) following the manufacturer's protocol.

\section{Statistical analysis}

Statistical analyses were performed using SigmaPlot ver. 9.0. Two-sided Student's t test was used when appropriate. The results are expressed as the mean \pm standard deviation or \pm standard error. A p-value less than 0.05 was considered to be statistically significant.

\section{Results}

\section{AZD1208 suppresses tumor growth in gastric cancer}

To determine the effects of AZD1208 treatment on human gastric cancer cell growth, we first treated each cell line with various concentrations of AZD1208 for 120 hours. Cell survival was then measured via the MTT assay (S2A Fig.). AZD1208 had a minimal anti-proliferative effect on most of the cell lines up to a concentration of $1 \mu \mathrm{M}$. At $10 \mu \mathrm{M}$, AZD1208 suppressed the proliferation of $\mathrm{N} 87$ and MKN45 cells by approximately $40 \%$; however, no obvious growth inhibi- 
<smiles>NC1CCCN(c2c(/C=C3\SC(=O)NC3=O)cccc2-c2ccccc2)C1</smiles>

Chemical formula: $\mathrm{C}_{21} \mathrm{H}_{21} \mathrm{~N}_{3} \mathrm{O}_{2} \mathrm{~S}$

Molecular weight: 379.48
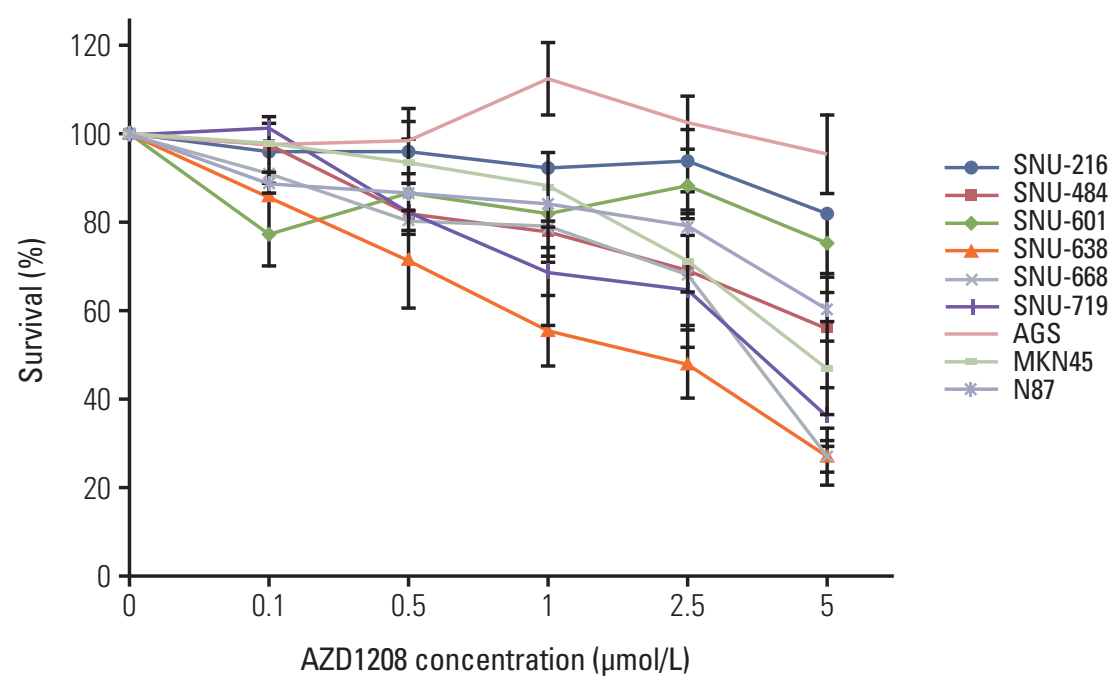

AZD1208

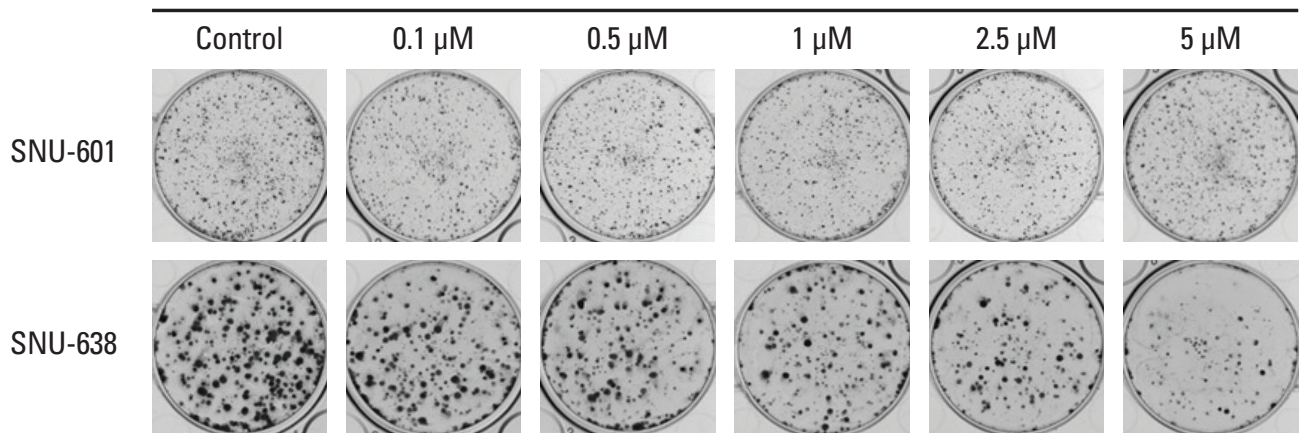

Fig. 1. Effects of AZD1208 treatment on the inhibition of human gastric cancer cell growth. (A) Chemical structure of AZD1208. (B) The growth inhibition effects of AZD1208 were evaluated with a colony formation assay. The percentage of surviving cells was calculated by counting the number of colonies and are shown as a graph with standard error bars ( $\mathrm{n}=3)$.

tion was observed during the early time points of the cell viability assay. Based on our data and previous reports stating that Pim kinases promote cell cycle progression and evasion of apoptosis signals, we had predicted a lower proliferation rate and active cell death signals when Pim kinases were disrupted $[21,22]$. We confirmed this prediction in longterm colony formation assays. We observed that AZD1208 treatment affected cell proliferation in gastric cancer cell lines (Fig. 1B, S2B Fig.), and several additional cell lines (i.e., SNU484, -638, and -719) showed greater dose-dependent sensi- 
Table 1. Effects of AZD1208 treatment on the inhibition of human gastric cancer cell growth in a colony CFA

\begin{tabular}{ll} 
Cell line & CFA: IC $_{50}(\mu \mathrm{mol} / \mathrm{L})$ \\
\hline SNU-216 & $>5$ \\
SNU-484 & $3.36 \pm 0.466$ \\
SNU-601 & $>5$ \\
SNU-638 & $1.47 \pm 0.082$ \\
SNU-668 & 3.10 \\
\hline SNU-719 & $3.11 \pm 1.47$ \\
AGS & $>5$ \\
MKN-45 & 4.69 \\
N87 & $>5$ \\
\hline
\end{tabular}

Values are presented as mean \pm standard deviation. CFA, colony formation assay.

tivity to AZD1208 treatment than other cell lines (Table 1); SNU-638 cells were the most sensitive to AZD1208 compared to other cell lines, and SNU-601 cells were the most resistant. Based on the results obtained, SNU-638 and SNU-601 cells were selected for further study. In addition, AZD1208 significantly delayed tumor growth in a SNU-638 xenograft model. The doubling time of tumor volume with vehicle treatment was 17 days, while the time to 2-fold increase of tumor volume with AZD1208 treatment was observed after 31 days, which supports the delay of tumor growth by AZD1208 treatment. Furthermore, AZD1208 treated mice showed lower Ki-67 expression, suggesting lower proliferation ability compared with non-treated mice. However, there was no meaningful increase in apoptosis in AZD1208 treated mice (S3 Fig.). These data demonstrated the antitumor effects of AZD1208 in a gastric cancer model.

\section{Expression of Pim isoforms is not correlated with the response to AZD1208}

To determine whether cell line sensitivity was correlated with Pim isoform expression, we analyzed the mRNA and protein expression levels of Pim-1, Pim-2, and Pim-3 in a panel of 10 gastric cancer cell lines. To assess mRNA expression, we performed RT-PCR and qRT-PCR analyses (S4 Fig.). Differential expression of Pim family members was observed, with Pim-3 expression mostly up-regulated as previously reported [5]. Additionally, we observed heterogeneous protein expression of the Pim isoforms in each cell line (Fig. 2). These data suggest that the basal levels of Pim-1, Pim-2, and Pim-3 mRNA and protein did not correlate with responses to AZD1208.

Interestingly however, we found that SNU-601 and AGS cells harboring PIK3CA mutations showed no sensitivity to AZD1208 (Table 2), raising the possibility that PI3K/Akt pathway activation may promote resistance to AZD1208.

\section{AZD1208 inhibits phosphorylation of Pim kinase sub- strates}

To determine whether AZD1208 was capable of inhibiting phosphorylation of proteins downstream of Pim kinases, we measured the phosphorylation and total protein expression levels of these downstream molecules by western blot analysis [1,3]. A significant dose-dependent reduction of phosphorylation of the Pim substrates Bad and 4E-BP1 in SNU-638 cells was observed. In addition, phosphorylation of S6K, another downstream effector of mammalian target of rapamycin complex 1 (mTORC1), was also markedly down-regulated. On the other hand, there were no changes in the levels of pro-survival proteins, such as Bcl-xl and Mcl-1. Moreover, we did not see any increase in the protein levels of PARP cleavage and caspase- 3 activity via immunoblotting or in

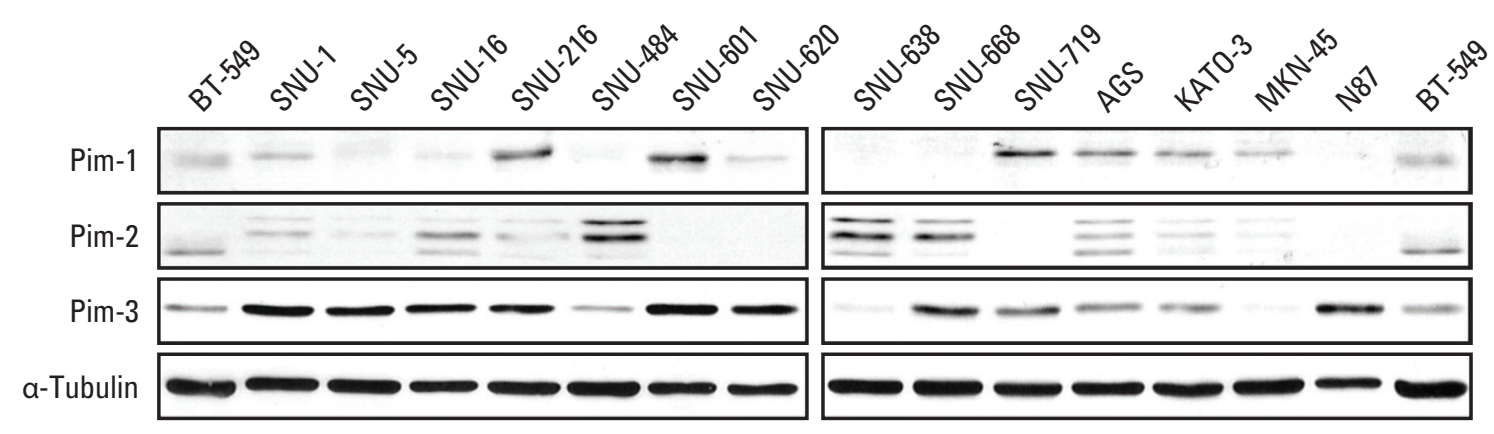

Fig. 2. Basal levels of Pim kinase protein expression in gastric cancer cell lines. Basal levels of Pim kinase proteins in human gastric cancer cell lines were confirmed by western blot analysis. BT-549 sample and $\alpha$-tubulin were used as loading controls. 
Table 2. Genetic background of gastric cancer cell lines

\begin{tabular}{|c|c|c|c|c|}
\hline Gastric cancer cell line & HER2 & PIKЗСА & TP53 & KRAS \\
\hline SNU-216 & Amp. & wt & $\mathrm{mt}$ & wt \\
\hline SNU-484 & Normal & wt & $\mathrm{mt}$ & wt \\
\hline SNU-601 & Normal & E542K & $\mathrm{mt}$ & $\mathrm{mt}$ \\
\hline SNU-638 & Normal & wt & $\mathrm{mt}$ & wt \\
\hline SNU-668 & Normal & wt & $\mathrm{mt}$ & $\mathrm{mt}$ \\
\hline SNU-719 & Normal & wt & wt & wt \\
\hline AGS & Normal & E453K & $\mathrm{mt}$ & wt \\
\hline MKN-45 & Normal & wt & wt & wt \\
\hline N87 & Amp. & wt & wt & wt \\
\hline
\end{tabular}

Amp, amplification; wt, wild type; mt, mutation.

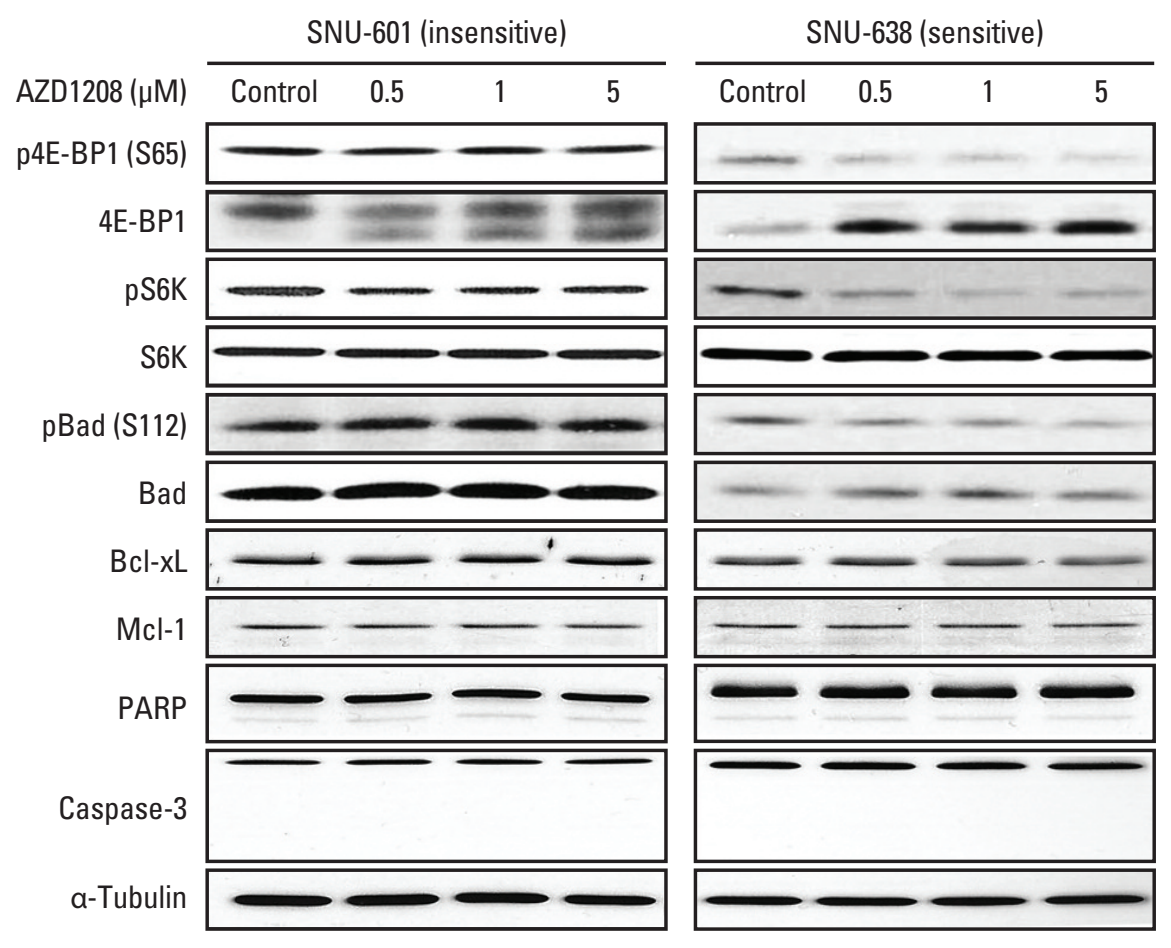

Fig. 3. Effects of AZD1208 treatment on the substrates of Pim kinase. Cells were treated with increasing doses of AZD1208 for 120 hours. Western blot analysis was performed with the indicated antibodies. $\alpha$-Tubulin was used as a loading control.

apoptotic cells via annexin V assays after AZD1208 treatment (Fig. 3, S5 Fig.).

Major proteins of oncogenic proliferation pathways, such as Akt or Erk, and modulators of Pim expression, such as the Jak/STAT pathway, were not affected by AZD1208 treatment in gastric cancer cells (data not shown). However, the influence of AZD1208 on downstream substrates of Pim kinase and downstream signals of mTORC1 correlated with drug sensitivity. Collectively, these data suggest that down- stream molecules of Pim kinase can be regulated by AZD1208 , but apoptosis induction or inhibition of proliferative signaling is not responsible for the anti-tumor effects of AZD1208.

\section{AZD1208 induces autophagic death of gastric cancer cells}

Since we found that AZD1208 did not induce apoptosis, we determined whether autophagy (or type II programmed 
A
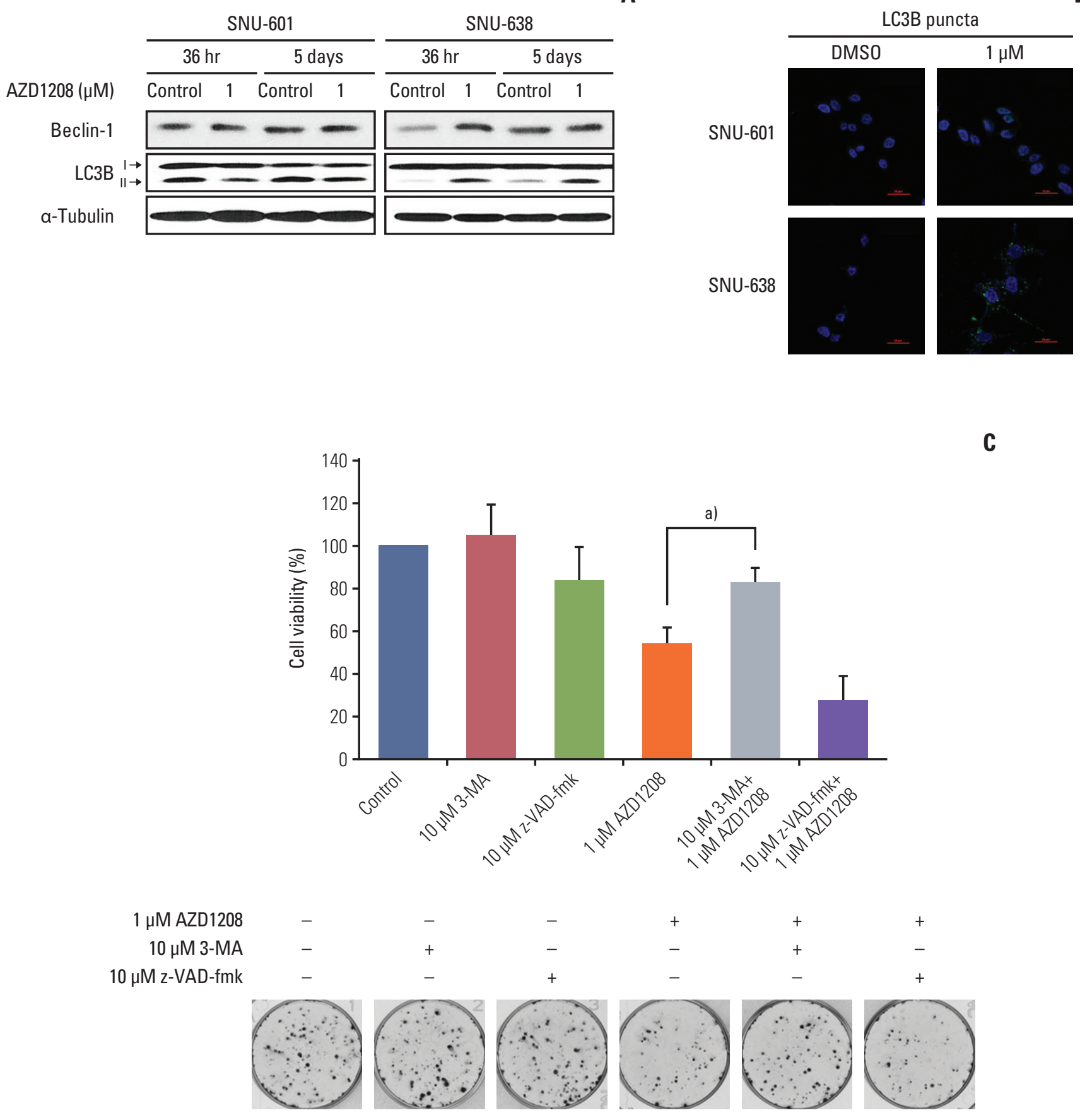

Fig. 4. Induction of cell death by AZD1208 through stimulation of autophagy. (A) Cells were treated with dimethyl sulfoxide (DMSO; control) or $1 \mu \mathrm{M}$ AZD1208 for 36 or 120 hours. The expression levels of light chain 3B (LC3B) and Beclin-1 were measured by western blot analysis. $\alpha$-Tubulin was used as a loading control. (B) SNU-601 and SNU-638 cells transfected with GFP-LC3B were treated with $1 \mu \mathrm{M}$ AZD1208 for 5 days. Confocal microscopy was used to observe the signals corresponding to LC3B expression (green fluorescence). DNA was counterstained with DAPI (blue). The merged images represent overlapping signals of the two channels. (C) SNU-638 cells were pre-treated with the autophagy inhibitor 3-methyladenine (3-MA; $10 \mu \mathrm{M})$ or caspase-3 inhibitor z-VAD-fmk $(10 \mu \mathrm{M})$ for 24 hours. Next, the cells were treated with $1 \mu \mathrm{M}$ AZD1208 every 3 days for 14 days. The percentages of surviving cells were calculated by counting the number of colonies and are presented in a bar graph with standard error bars $(n=3)$. a) $\mathrm{p}=0.008$. 


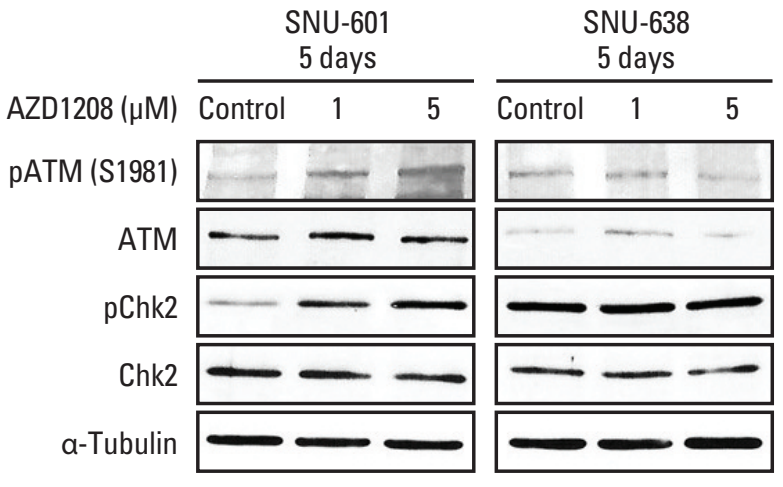

Fig. 5. Association of the DNA damage repair pathway with AZD1208 resistance. Cells were treated with dimethyl sulfoxide (control) and 1 or $5 \mu \mathrm{M}$ AZD1208 for 120 hours. The expression levels of ATM and Chk2 were measured by western blot analysis. $\alpha$-Tubulin was used as a loading control.

cell death) was related to the cytotoxic effects of AZD1208. First, we measured the expression levels of the well-known autophagy markers LC3B and Beclin-1 following AZD1208 treatment (Fig. 4A). LC3B is cleaved to form LC3B-II during autophagy; thus, conversion of LC3B-I to LC3B-II is associated with autophagosome formation. Interestingly, AZD1208 treatment induced up-regulation of LC3B-II expression at both time points in SNU-638 cells, but not in SNU-601 cells. By contrast, Beclin-1 expression was slightly up-regulated at 36 hours in SNU-638 cells, but no visible changes were observed after 5 days of exposure.

Next, we performed an immunofluorescence study to further confirm the role of AZD1208 on the induction of autophagy. Both SNU-601 and SNU-638 cells were transfected with a plasmid encoding GFP-tagged LC3B. Fluorescence microscopy was then performed to monitor GFP-tagged LC3 expression in both sensitive and resistant cells (Fig. 4B). SNU638 cells clearly showed re-localization of GFP-LC3B from a diffuse pattern into a punctate pattern corresponding to autophagosome formation following AZD1208 treatment. By contrast, GFP-LC3B localization was cytosolic and diffuse in SNU-601 cells, regardless of drug treatment.

To determine whether AZD1208 sensitivity was a direct result of autophagy and not apoptosis, we performed CFAs to measure the growth of SNU-638 cells in the presence of the autophagy inhibitor 3-MA and caspase inhibitor z-VADfmk (Fig. 4C). 3-MA treatment in SNU-638 cells effectively rescued the AZD1208-induced decrease in cell viability. However, z-VAD-fmk treatment did not result in restoration of cell viability but instead slightly decreased cell viability. These data demonstrate that autophagic cell death induced by AZD1208 has antitumor effects on gastric cancer cells.

\section{Regulation of the DNA damage response is associated with AZD1208 sensitivity}

A role of Pim kinases in repairing DNA damage has been reported [23]. We therefore determined whether AZD1208 can affect the DNA damage repair (DDR) pathway via western blot analysis. Intriguingly, ATM phosphorylation was up-regulated in a dose-dependent manner in insensitive SNU-601 cells along with Chk2 phosphorylation (Fig. 5). Consistent with these findings, we also observed that Chk2 expression was highly activated in the nuclei of SNU-601 cells, but not those of in SNU-638 cells (S6 Fig.). Data from these experiments revealed that AZD1208 treatment induced DNA damage and hyper-activation of the DDR in SNU-601 cells correlated with increased resistance to AZD1208. Depletion of Pim kinases can lead to DNA damage accumulation $[21,23]$, and regulation of the DDR may be related to drug sensitivity [24]. These results suggest that increased activity of the DDR system could be a mechanism underlying AZD1208 resistance.

\section{Combined treatment of AZD1208 with an Akt inhibitor enhances antitumor effects and overcomes drug resistance in gastric cancer cells}

Overactivation of the Akt signaling pathway has been detected in gastric cancer [25]. Pim can induce resistance to Akt inhibition, and Akt modulates DDR signaling through interactions with DNA damage sensors, such as ATM, ataxia telangiectasia and Rad3-related protein (ATR), as well as DNA-dependent protein kinase catalytic subunit [26]. Thus, we hypothesized that co-administration of Akt and Pim inhibitors may exert more potent cytotoxic effects than treatment with either reagent alone because the combination could block the compensatory actions between Akt and Pim and disrupt the DDR pathway. We therefore monitored the combined effects of Pim and Akt inhibition using CFAs. As expected, the percentage of growth inhibition for the gastric cancer cell lines observed with dual treatment was significantly higher than for treatment with each reagent alone (S7 Table). In particular, the colony formation ability of AZD1208-resistant SNU-601 cells was dramatically reduced by the combined treatment compared to exposure to AZD1208 alone (Fig. 6A).

To evaluate the signaling pathways involved in growth inhibition by combinatorial treatment with AZD1208 and Akt inhibitors, we examined the activities of 4E-BP1 and Bad, which are overlapping downstream molecules of the Pim and Akt cascades, respectively, in SNU-601 cells, in which synergistic effects were observed, and in SNU-668 cells, in which antagonistic effects were observed (Fig. 6B). We first confirmed the increased phosphorylation of Akt itself, which 

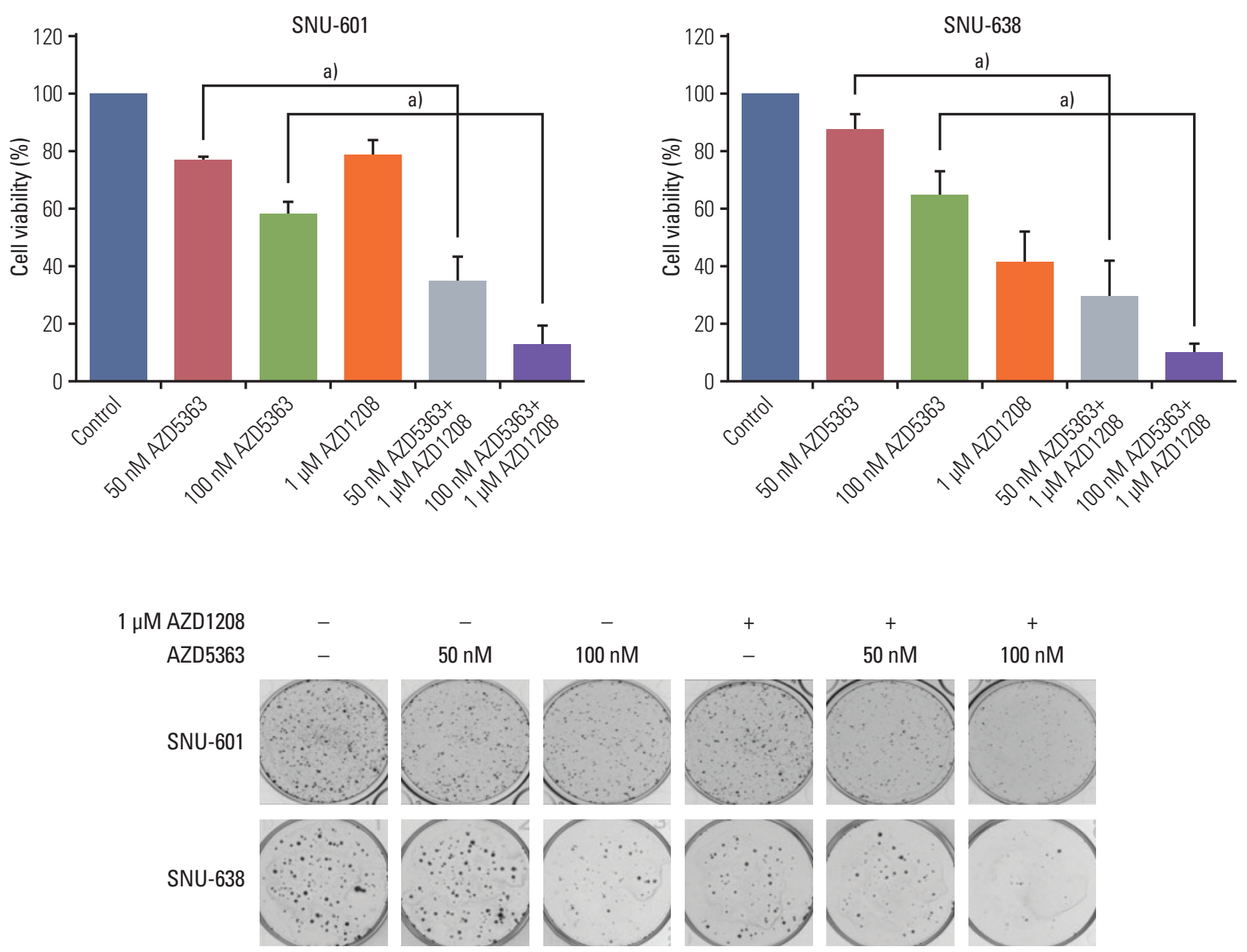

Fig. 6. Enhanced antitumor effects of the combination of AZD1208 and an Akt inhibitor in gastric cancer cells. (A) Cells were seeded and cultured with increasing concentrations of AZD5363 and $1 \mu \mathrm{M}$ AZD1208 every 3 days. The cells were cultured for 14 days until colonies formed and were then stained. The percentages of surviving cells were calculated by counting the number of colonies and are presented in a bar graph with standard error bars $(n=3)$. ${ }^{a} p<0.005$. (Continued to the next page)

is consistent with observations reported in previous studies [18]. Combined administration of AZD1208 and Akt inhibitors markedly decreased phosphorylation of 4E-BP1 and Bad kinase in comparison to either agent alone. In addition, phosphorylation of PRAS40 was significantly reduced only in SNU-601 cells, resulting in down-regulation of mTOR signaling activity. Furthermore, we observed that co-administration of AZD1208 and AZD5363 led to a significant reduction of pChk2 expression and increased the number of $\gamma-\mathrm{H} 2 \mathrm{AX}$ foci, a reasonable indicator of DNA double strand breaks, in SNU-601 cells (Fig. 6C). These results suggest that dual inhibition of Pim and Akt synergistically induce anti- cancer effects and could overcome resistance to AZD1208 through abrogation of DDR activity.

\section{Discussion}

Pim kinases are overexpressed in various types of tumors. Studies of the development of novel Pim kinase inhibitors have been published [27]. In a previous investigation, it was reported that AZD1208 is a potent pan-Pim kinase inhibitor 

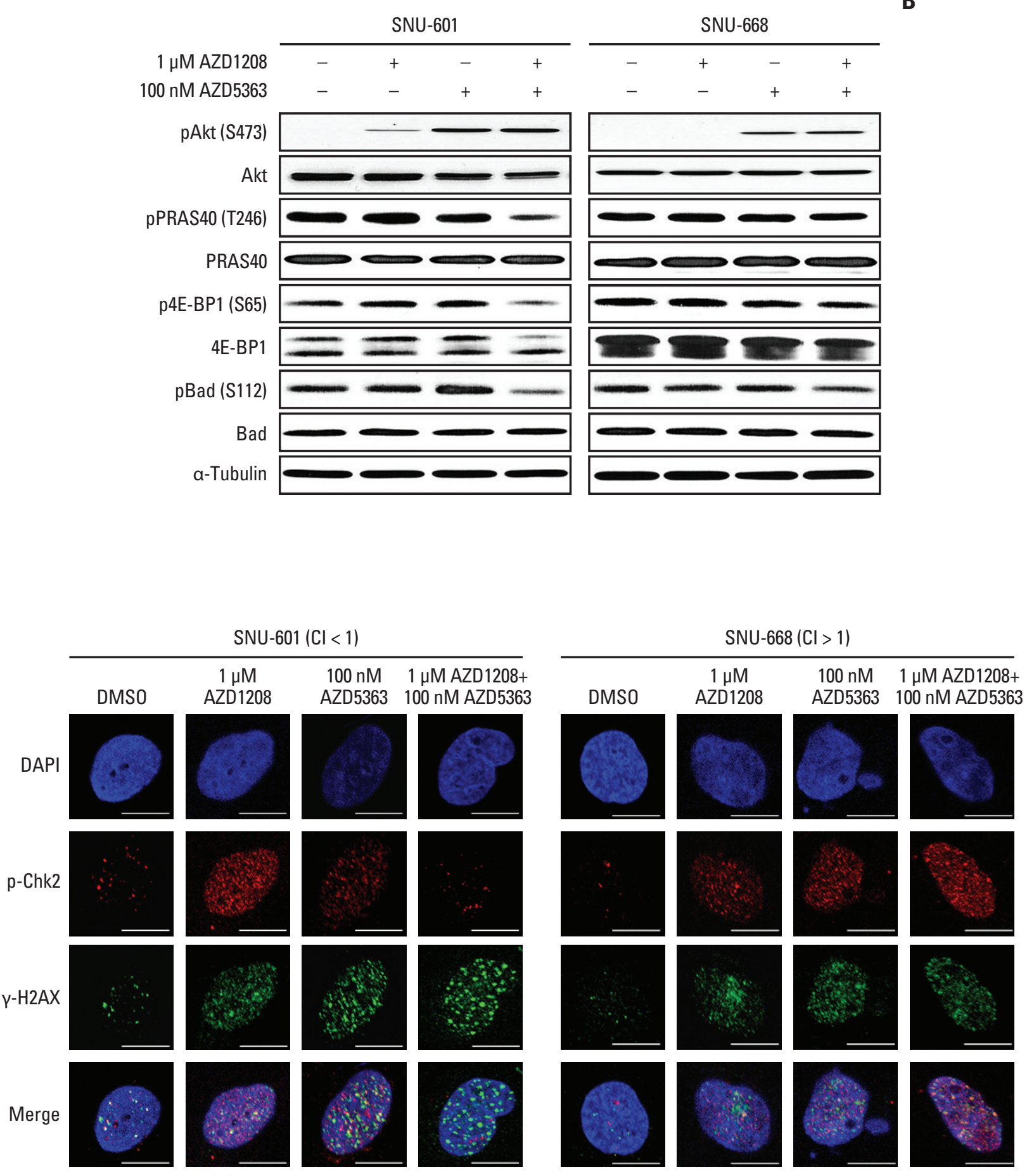

Fig. 6. (Continued from the previous page) (B) Cells were treated for 120 hours with $1 \mu$ M AZD1208 and/or $100 \mathrm{nM}$ AZD5363. The expression levels of the indicated proteins were determined by western blot analysis. $\alpha$-Tubulin was used as a loading control. (C) Cells were treated with AZD1208 and AZD5363 alone or in combination for 5 days, and immunofluorescence analysis was subsequently performed. Confocal microscopy was used to observe the signals corresponding to p-Chk2 (red) and $\gamma$-H2AX (green). DAPI (blue) was used as a nuclear counterstain. CI, combination index. Scale bars $=5 \mu \mathrm{m}$. 
with anti-tumor activity in acute myeloid leukemia [16], but the sensitivity of gastric cancer cell lines to Pim inhibition has not yet been assessed. In the present study, to our knowledge, we report the first assessment of the effects of AZD1208 and its underlying mechanisms of action in a gastric cancer cell line panel. AZD1208 down-regulated phosphorylation of Pim kinase substrates in a short period of time but did not induce apoptosis. Furthermore, AZD1208 reduced cell proliferation through autophagosome formation by LC3B in a long-term culture system. We also found that increased levels of DDR factors were associated with AZD1208 insensitivity. Moreover, we observed that the combination of AZD1208 with an Akt inhibitor had significant antitumor effects in gastric cancer cell lines, suggesting that while AZD1208 exerts therapeutic effects alone, these effects are more robust in combination with an Akt inhibitor.

Most studies of Pim inhibitors have shown that treatment with these reagents induces apoptosis through a reduction of phosphorylation of Pim kinase substrates [27]. However, we did not observe apoptotic cell death, although factors associated with cell survival, such as Bad and 4E-BP1, were down-regulated by Pim inhibition. On the other hand, it has been reported that the Pim inhibitor SGI-1776 induces limited apoptosis and autophagy in cases of multiple myeloma, although it was unclear how Pim inhibition caused autophagic cell death [28]. In our investigation, we determined that AZD1208 not only induced autophagic cell death but also attenuated DDR capacity. Wang et al. [21] noted that Pim-2 directly binds to and phosphorylates the cyclin-dependent kinase inhibitor p21 at Thr145. It is well-known that deregulated cell cycle progression causes replication stress and DNA damage [22]. Additionally, p53 induces autophagy in a damage-regulated autophagy modulator (DRAM)dependent manner in the presence of DNA damage [29]. Based on the results of these studies, Pim inhibition by AZD1208 led to the misregulation of intracellular signaling, resulting in increased DNA damage. Accumulation of DNA damage by Pim inhibition could also induce autophagy.

Activation of the ATM-Chk2 pathway was observed in SNU-601 cells following Pim inhibition. Interestingly, SNU601 cells were found to have low levels of DRAM mRNA expression, which affected the initiation of autophagy (data not shown). These findings suggest that the DNA damage induced by AZD1208 is efficiently repaired, resulting in resistance to AZD1208.

Targeting the Akt signaling pathway has produced a limited antitumor effect to date due to drug resistance through a negative feedback loop as well as toxicity $[14,30]$. The present study is the first to report that inhibition of Pim could overcome these problems and induce cell death to a greater extent in gastric cancer cells. Moreover, the findings of the present study provide the first evidence that AZD1208 syn- ergistically enhances AZD5363-mediated growth inhibitory effects as well as inhibits 4E-BP1 and Bad phosphorylation in most gastric cancer cell lines, which suggests that Pim inhibitors can act as sensitizers for Akt inhibitors in gastric cancer cell lines. Additionally, the synergistic effects of Pim and Akt co-targeting are attributable to decreased DDR capacity, which is a major mechanism of AZD1208 resistance, resulting in increased cellular sensitivity to AZD1208. These results provide a rationale for administering Pim and Akt inhibitors in combination to treat gastric cancer.

The findings in our study suggest that Pim inhibition, synergistically with Akt inhibition, can be a potential strategy for treating gastric cancer. Furthermore, our findings provide significant insights into the mechanism underlying the effects of Pim inhibition.

\section{Electronic Supplementary Material}

Supplementary materials are available at Cancer Research and Treatment website (https://www.e-crt.org).

\section{Conflicts of Interest}

Yung-Jue Bang has advised or consulted for and received academic research funding from Bayer, Novartis, Boehringer-Ingelheim, Roche/Genentech, AstraZeneca, Merck Serano, MSD, BristolMyers Squibb, Eli Lilly, Pfizer, ONO, Taiho, and GreenCross. SeockAh Im is a recipient of academic research funds from AstraZeneca, Inc., and has served as a consultant and on the advisory board for AstraZeneca, Novartis, and Roche without compensation. Do-Youn $\mathrm{Oh}$ is a recipient of academic research funds from AstraZeneca, Inc. None of the other authors declare any potential conflicts of interest.

\section{Acknowledgments}

We thank Dennis Huszar (AstraZeneca Oncology, Waltham, MA) and Barry Davies (AstraZeneca Oncology, Alderly Park, Macclesfield, UK) for providing AZD1208 and AZD5363, respectively.

This work was supported by the Priority Research Centers Program through the National Research Foundation of Korea (NRF) funded by the Ministry of Education, Science and Technology (20090093820) 


\section{References}

1. Narlik-Grassow M, Blanco-Aparicio C, Carnero A. The PIM family of serine/threonine kinases in cancer. Med Res Rev. 2014;34:136-59.

2. Brault L, Gasser C, Bracher F, Huber K, Knapp S, Schwaller J. $\mathrm{PIM}$ serine/threonine kinases in the pathogenesis and therapy of hematologic malignancies and solid cancers. Haematologica. 2010;95:1004-15.

3. Nawijn MC, Alendar A, Berns A. For better or for worse: the role of Pim oncogenes in tumorigenesis. Nat Rev Cancer. 2011;11:23-34.

4. Warnecke-Eberz U, Bollschweiler E, Drebber U, Metzger R, Baldus SE, Holscher AH, et al. Prognostic impact of protein overexpression of the proto-oncogene PIM-1 in gastric cancer. Anticancer Res. 2009;29:4451-5.

5. Zheng HC, Tsuneyama K, Takahashi H, Miwa S, Sugiyama T, Popivanova BK, et al. Aberrant Pim-3 expression is involved in gastric adenoma-adenocarcinoma sequence and cancer progression. J Cancer Res Clin Oncol. 2008;134:481-8.

6. Khanna P, Chua PJ, Bay BH, Baeg GH. The JAK/STAT signaling cascade in gastric carcinoma (Review). Int J Oncol. 2015; 47:1617-26.

7. Vivanco I, Sawyers CL. The phosphatidylinositol 3-Kinase AKT pathway in human cancer. Nat Rev Cancer. 2002;2:489501.

8. LoPiccolo J, Blumenthal GM, Bernstein WB, Dennis PA. Targeting the PI3K/Akt/mTOR pathway: effective combinations and clinical considerations. Drug Resist Updat. 2008;11:32-50.

9. Chandarlapaty S, Sawai A, Scaltriti M, Rodrik-Outmezguine V, Grbovic-Huezo O, Serra V, et al. AKT inhibition relieves feedback suppression of receptor tyrosine kinase expression and activity. Cancer Cell. 2011;19:58-71.

10. Muranen T, Selfors LM, Worster DT, Iwanicki MP, Song L, Morales FC, et al. Inhibition of PI3K/ mTOR leads to adaptive resistance in matrix-attached cancer cells. Cancer Cell. 2012;21: 227-39.

11. Altomare DA, Khaled AR. Homeostasis and the importance for a balance between AKT/mTOR activity and intracellular signaling. Curr Med Chem. 2012;19:3748-62.

12. Amaravadi R, Thompson CB. The survival kinases Akt and Pim as potential pharmacological targets. J Clin Invest. 2005; 115:2618-24.

13. Hammerman PS, Fox CJ, Birnbaum MJ, Thompson CB. Pim and Akt oncogenes are independent regulators of hematopoietic cell growth and survival. Blood. 2005;105:4477-83.

14. Cen B, Mahajan S, Wang W, Kraft AS. Elevation of receptor tyrosine kinases by small molecule AKT inhibitors in prostate cancer is mediated by Pim-1. Cancer Res. 2013;73:3402-11.

15. Meja K, Stengel C, Sellar R, Huszar D, Davies BR, Gale RE, et al. PIM and AKT kinase inhibitors show synergistic cytotoxicity in acute myeloid leukaemia that is associated with convergence on mTOR and MCL1 pathways. Br J Haematol. 2014;167:69-79.
16. Keeton EK, McEachern K, Dillman KS, Palakurthi S, Cao Y, Grondine MR, et al. AZD1208, a potent and selective pan-Pim kinase inhibitor, demonstrates efficacy in preclinical models of acute myeloid leukemia. Blood. 2014;123:905-13.

17. Dakin LA, Block MH, Chen H, Code E, Dowling JE, Feng X, et al. Discovery of novel benzylidene-1,3-thiazolidine-2,4-diones as potent and selective inhibitors of the PIM-1, PIM-2, and PIM-3 protein kinases. Bioorg Med Chem Lett. 2012;22:4599604.

18. Davies BR, Greenwood H, Dudley P, Crafter C, Yu DH, Zhang J, et al. Preclinical pharmacology of AZD5363, an inhibitor of AKT: pharmacodynamics, antitumor activity, and correlation of monotherapy activity with genetic background. Mol Cancer Ther. 2012;11:873-87.

19. Ku JL, Park JG. Biology of SNU cell lines. Cancer Res Treat. 2005;37:1-19.

20. Kang S, Min A, Im SA, Song SH, Kim SG, Kim HA, et al. TGFbeta suppresses COX-2 expression by tristetraprolin-mediated RNA destabilization in A549 human lung cancer cells. Cancer Res Treat. 2015;47:101-9.

21. Wang Z, Zhang Y, Gu JJ, Davitt C, Reeves R, Magnuson NS. Pim-2 phosphorylation of p21(Cip1/WAF1) enhances its stability and inhibits cell proliferation in HCT116 cells. Int J Biochem Cell Biol. 2010;42:1030-8.

22. Bartek J, Lukas J. DNA damage checkpoints: from initiation to recovery or adaptation. Curr Opin Cell Biol. 2007;19:238-45.

23. Zirkin S, Davidovich A, Don J. The PIM-2 kinase is an essential component of the ultraviolet damage response that acts upstream to E2F-1 and ATM. J Biol Chem. 2013;288:21770-83.

24. Helleday T, Petermann E, Lundin C, Hodgson B, Sharma RA. DNA repair pathways as targets for cancer therapy. Nat Rev Cancer. 2008;8:193-204.

25. Ang KL, Shi DL, Keong WW, Epstein RJ. Upregulated Akt signaling adjacent to gastric cancers: implications for screening and chemoprevention. Cancer Lett. 2005;225:53-9.

26. Xu N, Lao Y, Zhang Y, Gillespie DA. Akt: a double-edged sword in cell proliferation and genome stability. J Oncol. 2012;2012:951724.

27. Blanco-Aparicio C, Collazo AM, Oyarzabal J, Leal JF, Albaran MI, Lima FR, et al. Pim 1 kinase inhibitor ETP-45299 suppresses cellular proliferation and synergizes with PI3K inhibition. Cancer Lett. 2011;300:145-53.

28. Cervantes-Gomez F, Chen LS, Orlowski RZ, Gandhi V. Biological effects of the Pim kinase inhibitor, SGI-1776, in multiple myeloma. Clin Lymphoma Myeloma Leuk. 2013;13 Suppl 2:S317-29.

29. Crighton D, Wilkinson S, O'Prey J, Syed N, Smith P, Harrison PR, et al. DRAM, a p53-induced modulator of autophagy, is critical for apoptosis. Cell. 2006;126:121-34.

30. Fruman DA, Rommel C. PI3K and cancer: lessons, challenges and opportunities. Nat Rev Drug Discov. 2014;13:140-56. 